\title{
Letter: The effects of streamwise system rotation on pressure fluctuations in a turbulent channel flow
}

Cite as: Phys. Fluids 30, 091701 (2018); https://doi.org/10.1063/1.5048800

Submitted: 17 July 2018 . Accepted: 28 August 2018 . Published Online: 11 September 2018

Zixuan Yang (D), Bing-Qing Deng, Bing-Chen Wang, and Lian Shen
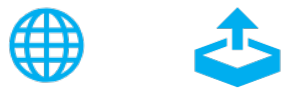

\section{ARTICLES YOU MAY BE INTERESTED IN}

Letter: Hydroelastic interactions between water waves and floating freshwater ice Physics of Fluids 30, 091702 (2018); https://doi.org/10.1063/1.5050262

Study of the thrust-drag balance with a swimming robotic fish

Physics of Fluids 30, 091901 (2018); https://doi.org/10.1063/1.5043137

Letter: An origin of magnetohydrodynamic reverse flow in $90^{\circ}$ bends

Physics of Fluids 30, 081701 (2018); https://doi.org/10.1063/1.5046328

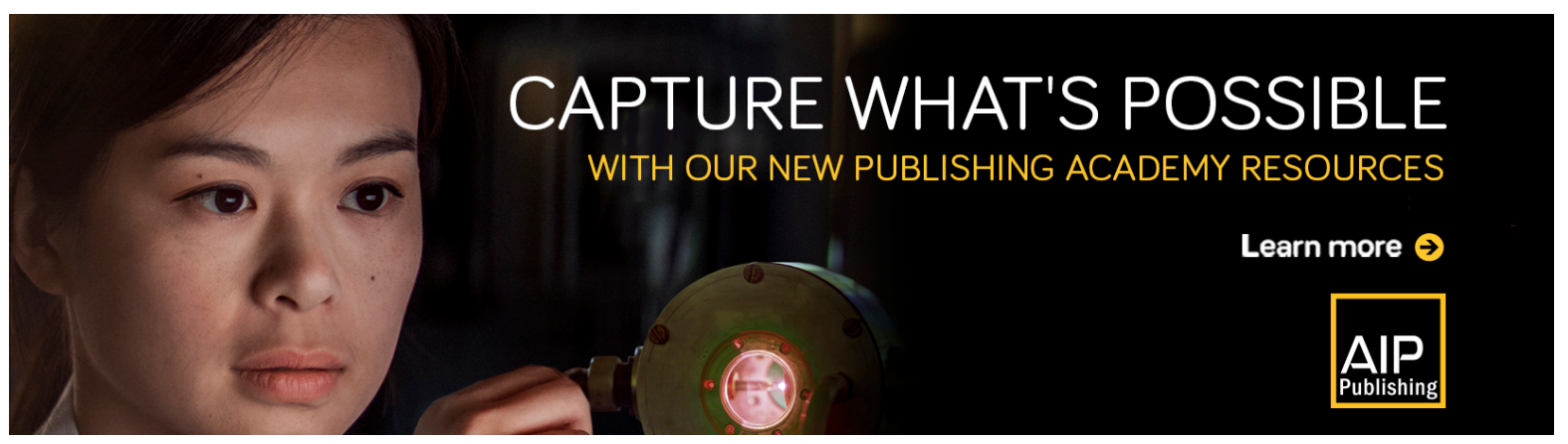




\title{
Letter: The effects of streamwise system rotation on pressure fluctuations in a turbulent channel flow
}

\author{
Zixuan Yang, ${ }^{1,2}$ Bing-Qing Deng, ${ }^{2}$ Bing-Chen Wang, ${ }^{3, a)}$ and Lian Shen ${ }^{2}$ \\ ${ }^{1}$ State Key Laboratory of Nonlinear Mechanics, Institute of Mechanics, Chinese Academy of Sciences, \\ Beijing 100190, China \\ ${ }^{2}$ Department of Mechanical Engineering and St. Anthony Falls Laboratory, University of Minnesota, \\ Minneapolis, Minnesota 55455, USA \\ ${ }^{3}$ Department of Mechanical Engineering, University of Manitoba, Winnipeg, Manitoba R3T 5V6, Canada
}

(Received 17 July 2018; accepted 28 August 2018; published online 11 September 2018)

\begin{abstract}
In this letter, we report the modulating effects of streamwise system rotation on both the amplitude and the wavenumber of pressure fluctuations in a plane channel flow. The analysis of the pressure field is conducted based on a set of comprehensive direct numerical simulation data of six rotation numbers. It is observed that high pressure fluctuation regions collocate with the Taylor-Görtler-like (TGL) vortex cores. By decomposing the pressure field into rotation-induced and convection-induced parts, it is observed that the rotation-induced part dominates the total pressure fluctuations and facilitates the growth of TGL vortices. Furthermore, through a spectral analysis, it is discovered that the system rotation acts as a "linear amplifier," which converts high-wavenumber low-amplitude streamwise vorticity fluctuations into low-wavenumber high-amplitude pressure fluctuations. Published by AIP Publishing. https://doi.org/10.1063/1.5048800
\end{abstract}

Pressure-driven turbulent flow between two infinitely large plates subjected to a streamwise system rotation represents a geometrically simple but physically complex problem due to the coexistence of the mean and turbulent large-scale secondary flows. The primary (or mean) mode of secondary flows in the form of the so-called "double S-shaped triplezero-crossing patterned" mean spanwise flow has been well studied in the literature. ${ }^{1-6}$ Based on their systematic direct numerical simulation (DNS) study, Yang and Wang ${ }^{7}$ recently reported the second (or turbulent) mode of the secondary flows, referred to as Taylor-Görtler-like (TGL) vortices, occurring as two-layer streamwise-elongated counter-rotating roll cells. The streamwise scale of TGL vortices grows rapidly with an increasing rotation number, while the spanwise scale of TGL vortices remains stable due to the restriction from the channel height. In fluid mechanics, Taylor-Görtler vortices typically refer to the secondary flow structures induced by the centrifugal instability in a boundary layer flow over a concave surface. ${ }^{8,9}$ Analogous to the centrifugal instability, TGL vortices induced by the Coriolis force instability are observed in both spanwise- and streamwise-rotating channel flows. ${ }^{7,10,11}$ The existence of TGL vortices is critical for momentum transfer and influences significantly the flow statistics in both physical and spectral spaces. However, it is not clear why the streamwise wavelength of TGL vortices increases drastically as the rotation number increases in a streamwise-rotating channel flow. ${ }^{7}$

As a continuation of our previous research, ${ }^{7}$ in this letter, we report the finding of modulating effects of streamwise system rotation on both the amplitude and the wavenumber

a)Electronic mail: BingChen.Wang@Umanitoba.Ca of pressure fluctuations in a turbulent plane channel flow. We demonstrate that the pressure field significantly influences the size, strength, and characteristic wavelength of TGL vortices in a fast streamwise-rotating flow. This mechanism underlying the TGL vortices in a fast streamwise-rotating flow is essentially different from that for hairpin structures in wallshear turbulence because the hairpin structures are dominated by the strong shear instead of the pressure of a wall-bounded turbulent flow. ${ }^{12-14}$

Figure 1 shows the computational domain and coordinate system for performing DNS of a streamwise-rotating channel flow. As shown in the figure, $x_{1}, x_{2}$, and $x_{3}$ denote the streamwise, wall-normal, and spanwise coordinates, respectively, and $u_{1}, u_{2}$, and $u_{3}$ represent the velocity components in the corresponding directions. The continuity and momentum equations for an incompressible flow subjected to a streamwise system rotation take the following form:

$$
\begin{gathered}
\frac{\partial u_{i}}{\partial x_{i}}=0, \\
\frac{\partial u_{i}}{\partial t}+u_{j} \frac{\partial u_{i}}{\partial x_{j}}=-\frac{1}{\rho} \frac{\partial p}{\partial x_{i}}+v \frac{\partial^{2} u_{i}}{\partial x_{j} \partial x_{j}}-2 \varepsilon_{i 1 j} \Omega u_{j}-\frac{\Pi}{\rho} \delta_{i 1},
\end{gathered}
$$

where $p$ represents the effective pressure, $\Omega$ is the angular velocity of the system rotation, $\varepsilon_{i j k}$ is the Levi-Civita symbol, $\Pi$ is a constant streamwise pressure gradient that drives the flow, and $\delta_{i j}$ denotes the Kronecker delta. No-slip boundary conditions are imposed on the two solid walls, and periodic boundary conditions are applied to the streamwise and spanwise directions.

The new findings are obtained by analyzing the DNS database established by Yang and Wang, ${ }^{7}$ which involves six different rotation numbers: $R o_{\tau}=2 \Omega h / u_{\tau}=0,7.5,15,30,75$, 


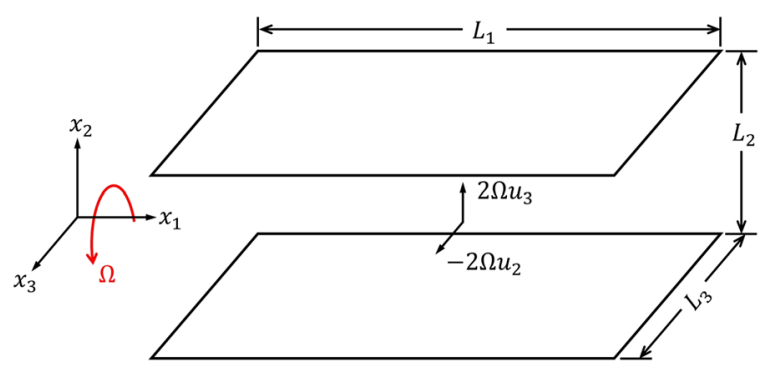

FIG. 1. Computational domain for a streamwise-rotating channel flow.

and 150, where $h$ represents one-half of the channel height and $u_{\tau}$ is the wall friction velocity. The highest rotation number $\left(R o_{\tau}=150\right)$ analyzed here is also the highest for streamwiserotating flows in the current literature, which facilitates this study of the effects of streamwise system rotation on the modulation of the pressure field and growth of TGL vortices. Before the establishment of this comprehensive DNS dataset, ${ }^{7}$ the highest rotation number in the literature was only $R o_{\tau}=30$, reported by Yang, Su, and $\mathrm{Wu}^{6}$ in 2010 . A very long computational domain of $L_{1} \times L_{2} \times L_{3}=512 \pi h \times 2 h \times 8 \pi h$ (with $16384 \times 128 \times 256$ grid points) has been used to perform DNS at $R o_{\tau}=150$ in order to capture the streamwise-elongated vortex structures. The Reynolds number is fixed at $R e_{\tau}=u_{\tau} h / v$ $=180$ in all cases. In presenting the results, we use a pair of angular brackets $\langle\cdot\rangle$ to denote temporal- and plane-averaging, and subsequently, the fluctuating component of an arbitrary variable $\phi$ is determined as $\phi^{\prime}=\phi-\langle\phi\rangle$.

Figure 2 shows typical vortex structures at $R o_{\tau}=150$ in both cross-stream $\left(x_{2}-x_{3}\right)$ and horizontal $\left(x_{1}-x_{3}\right)$ planes. To make the figure readable, only a small portion of the spanwise computational domain is shown in Fig. 2(a) and 1/32 of the streamwise computational domain is shown in Fig. 2(b). Because the TGL vortices are elongated in the streamwise direction, the instantaneous vortex structures extracted from different cross-stream planes feature common statistical characteristics. As such, to demonstrate the instantaneous TGL vortex structures, we arbitrarily choose the cross-stream plane located at $x_{1} / h=0$ in Fig. 2(a). From Fig. 2(a), it is interesting to observe that positive and negative pressure fluctuations $p^{\prime}$ collocate with the large-scale vortices rotating in the counterclockwise and clockwise directions, respectively. Figure 2(b) shows that the TGL vortices visualized using the contours of instantaneous pressure fluctuations $p^{\prime}$ are elongated in the streamwise direction. The streamwise and spanwise scales of TGL vortices reach $4.8 \times 10^{4}$ and 430 wall units, respectively, at $R o_{\tau}=150$.
In order to further study the effect of system rotation on the pressure field, we take the divergence of Eq. (2) and apply the divergence-free condition (1) to obtain the following Poisson equation for pressure, viz.:

$$
\frac{1}{\rho} \frac{\partial^{2} p}{\partial x_{i} \partial x_{i}}=-\frac{\partial u_{i}}{\partial x_{j}} \frac{\partial u_{j}}{\partial x_{i}}+2 \Omega \omega_{1},
$$

where $\omega_{1} \stackrel{\text { def }}{=} \partial u_{3} / \partial x_{2}-\partial u_{2} / \partial x_{3}$ is the streamwise vorticity. At the two walls, Eq. (2) degenerates to

$$
\frac{\partial p}{\partial x_{2}}=\rho v \frac{\partial^{2} u_{2}}{\partial x_{2}^{2}} \quad \text { at } \quad x_{2}= \pm h,
$$

which is the boundary condition for the pressure field. Pressure $p$ can be further decomposed into a rotation-induced component $p_{r}$ and a convection-induced component $p_{c}$, governed by the following two Poisson equations, respectively:

$$
\begin{gathered}
\frac{1}{\rho} \frac{\partial^{2} p_{r}}{\partial x_{i} \partial x_{i}}=2 \Omega \omega_{1}, \\
\frac{1}{\rho} \frac{\partial^{2} p_{c}}{\partial x_{i} \partial x_{i}}=-\frac{\partial u_{i}}{\partial x_{j}} \frac{\partial u_{j}}{\partial x_{i}} .
\end{gathered}
$$

Correspondingly, the boundary condition (4) can be decomposed into

$$
\left.\begin{array}{l}
\frac{\partial p_{r}}{\partial x_{2}}=0 \\
\frac{\partial p_{c}}{\partial x_{2}}=\rho v \frac{\partial^{2} u_{2}}{\partial x_{2}^{2}}
\end{array}\right\} \quad \text { at } \quad x_{2}= \pm h
$$

Because Eqs. (5)-(7) are linear with respect to pressure, $p \equiv p_{r}+p_{c}$ holds strictly. There is an additional constraint on the rotation-induced pressure $p_{r}$ : in the context of a nonrotating flow $\left(R o_{\tau}=0\right), p_{r}$ must remain constant throughout the computational domain such that $\partial p_{r} / \partial x_{i} \equiv 0$. With this additional condition, it is required that the boundary condition $\left(\partial p_{r} / \partial x_{2}\right)_{x_{2}= \pm h} \equiv 0$ must hold. Otherwise, $p_{r}$ would have a non-constant distribution and would influence the flow field in a non-rotating channel, which is an unphysical conclusion. In other words, by definition, the rotation-induced pressure $p_{r}$ must not have an impact on the flow in a non-rotating channel. In view of this, the decomposition of the boundary condition (7) is unique.

A fundamental difference between a streamwise-rotating flow and a non-rotating flow lies in the Poisson equation system that governs the pressure field. From the above linear decomposition of the pressure, it is clear that in a non-rotating flow, the pressure field is governed by just one Poisson equation (6) through the convection mechanism only, whereas in a
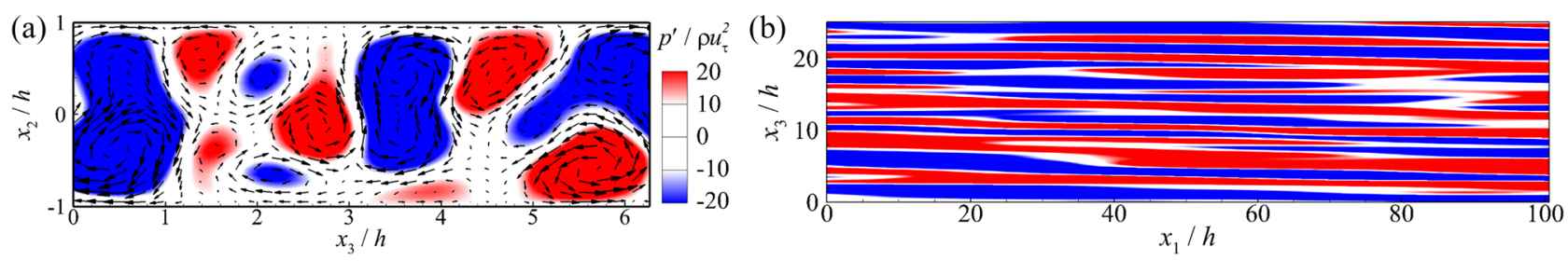

FIG. 2. TGL vortices visualized using the contours of pressure fluctuation $p^{\prime}$ at $R o_{\tau}=150$ in (a) the $x_{2}-x_{3}$ plane located at $x_{1} / h=0$ and (b) the $x_{1}-x_{3}$ plane located at $x_{2} / h=-0.5$. Vectors consisting of $u_{2}^{\prime}$ and $u_{3}^{\prime}$ are superimposed in panel (a) to show the rotating direction of vortices. 


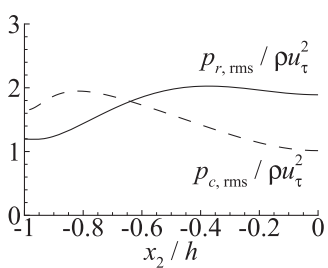

(a)

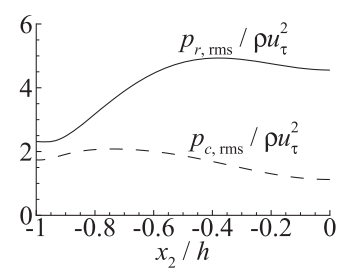

(b)

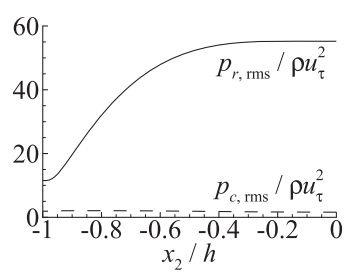

(c)
FIG. 3. Profiles of $p_{r, \mathrm{rms}}$ and $p_{c, \mathrm{rms}}$ at (a) $R o_{\tau}=7.5$, (b) $R o_{\tau}=15$, and (c) $R o_{\tau}=150$. streamwise-rotating flow, the total pressure field is governed jointly by two Poisson equations (5) and (6) through both the rotation and the convection mechanisms. Both Eqs. (5) and (6) can be solved analytically. Later, it will be shown that it is the rotation-induced pressure field governed by Eq. (5) that facilitates the growth of TGL vortices and plays the dominant role in a streamwise-rotating turbulent plane channel flow. It should be further indicated here that only the instantaneous pressure $p$ needs to be solved in DNS, and Eqs. (3)-(7) can be solved during post-processing of the DNS data obtained.

Figure 3 compares the profiles of the root-mean-square (RMS) of pressure fluctuations $p_{r, \mathrm{rms}}$ and $p_{c, \mathrm{rms}}$ at $R o_{\tau}=7.5$, 15 , and 150 . Because both $p_{r, \mathrm{rms}}$ and $p_{c, \mathrm{rms}}$ are symmetric about the channel center $\left(x_{2}=0\right)$, we only show their profiles within the lower half of the channel. As shown in Fig. 3(a), at $R o_{\tau}=7.5$, the magnitude of $p_{r, \mathrm{rms}}$ is comparable to that of $p_{c, \mathrm{rms}}$. In fact, $p_{r, \mathrm{rms}} \leq p_{c, \mathrm{rms}}$ holds only if $x_{2} / h \leq 0.64$. However, as is evident from Fig. 3(b), as the rotation number increases to $R o_{\tau}=15$, the magnitude of $p_{r, \mathrm{rms}}$ becomes larger than that of $p_{c, \text { rms }}$ across the entire channel. Finally, as the rotation number further increases to $R o_{\tau}=150$, the magnitude of $p_{r, \text { rms }}$ becomes significantly larger than that of $p_{c, \text { rms }}$, indicating an absolute dominance of rotation-induced pressure fluctuations over convection-induced pressure fluctuations (at a very high rotation number). In order to further understand this dominant effect of $p_{r, \text { rms }}$, Figs. 4(a) and 4(b) compare the profiles of $\omega_{1, \text { rms }}$ and $p_{r, \text { rms }}$ at various rotation numbers, respectively. As shown in Fig. 4(a), the effect of $R o_{\tau}$ on the magnitude of $\omega_{1, \mathrm{rms}}$ is non-monotonic as the trend reverses at $R o_{\tau}=15$. Although the profile of $\omega_{1, \text { rms }}$ varies slightly with $R o_{\tau}$ around $x_{2} / h=0.9$, in general, the magnitude of $\omega_{1, \mathrm{rms}}$ is insensitive to $R o_{\tau}$. By contrast, as shown in Fig. 4(b), the effect of system rotation on the rotation-induced pressure fluctuations is drastic, and specifically, the magnitude of $p_{r, \mathrm{rms}}$ increases monotonically with an increasing $R o_{\tau}$ value.

By comparing Figs. 4(a) and 4(b), it is clear that the rotation effects on the fluctuations of $\omega_{1}$ and $p_{r}$ are different.
However, from Eq. (5), it is understood that the instantaneous values of $p_{r}$ and $\omega_{1}$ are connected linearly through a Poisson equation. Because both Eq. (5) and the boundary condition for $p_{r}$ [the first condition of Eq. (7)] are linear, the same equation and boundary condition hold for $p_{r}^{\prime}$, i.e., $\partial^{2} p_{r}^{\prime} / \partial x_{i} \partial x_{i}=2 \rho \Omega \omega_{1}^{\prime}$ with $\partial p_{r}^{\prime} / \partial x_{2}=0$ at $x_{2}= \pm h$. Clearly, the role of system rotation (as indicated by $\Omega$ ) is to linearly amplify the conversion of the streamwise vorticity fluctuations $\omega_{1}^{\prime}$ into the rotation-induced pressure fluctuations $p_{r}^{\prime}$. In other words, large-scale streamwise vorticity fluctuations $\left(\omega_{1}^{\prime}\right)$ enter the source term (i.e., $\left.2 \rho \Omega \omega_{1}^{\prime}\right)$ of the Poisson equation, which "powers" rotation-induced pressure fluctuations $\left(p_{r}^{\prime}\right)$. Because the magnitude of $\omega_{1}^{\prime}$ is insensitive to $R o_{\tau}$, the value of $p_{r, \text { rms }}$ grows almost linearly with respect to $R o_{\tau}$. In consequence, the pressure fluctuations shown in Fig. 3(c) are dominated by $p_{r, \text { rms }}$ at a very high rotation number $R o_{\tau}=150$. As is clear from Eq. (3), the influences of convection and system rotation on the pressure field are independent of each other. Therefore, it is expected that the effect of system rotation on flow dynamics and structures enhances as the rotation number increases. Furthermore, based on the study of non-rotating channel flows, ${ }^{15}$ it is understood that the magnitude of $p_{c, \text { rms }} / \rho u_{\tau}^{2}$ tends to be insensitive to the Reynolds number. The turbulence flow structures induced by convection and system rotation are very different in terms of their length scales, which can be quantified through the following spectral analysis.

The system rotation modulates not only the amplitude but also the wavenumber of pressure fluctuations. The modulation effect on the amplitude of $p_{r}^{\prime}$ has been well demonstrated in Fig. 4(b). In order to further understand the modulation effects on the wavenumber of $p_{r}^{\prime}$, a spectral analysis is necessary. Performing the Fourier transform on Eq. (5) over the $x_{1}-x_{3}$ plane yields

$$
\frac{1}{\rho}\left(-k_{1}^{2}-k_{3}^{2}+\frac{\mathrm{d}^{2}}{\mathrm{~d} x_{2}^{2}}\right) \hat{p}_{r}=2 \Omega \hat{\omega}_{1} .
$$

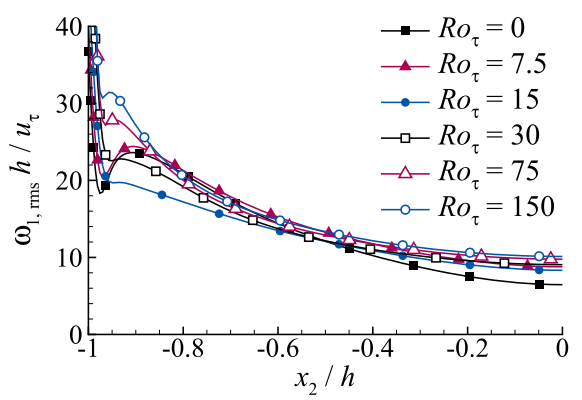

(a)

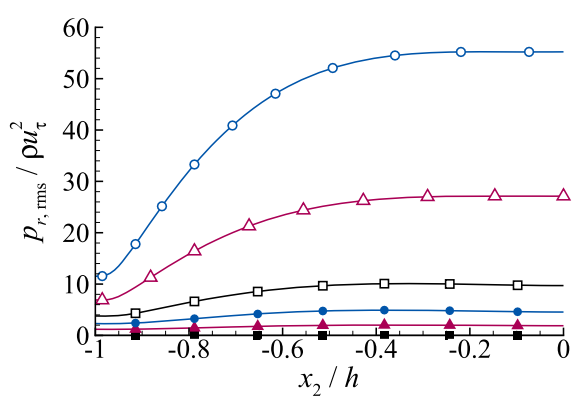

(b)
FIG. 4. Profiles of (a) $\omega_{1, \text { rms }}$ and (b) $p_{r, \mathrm{rms}}$ at various rotation numbers. 
Here, $\hat{p}_{r}=\hat{p}_{r}\left(k_{1}, x_{2}, k_{3}\right)$ and $\hat{\omega}_{1}=\hat{\omega}\left(k_{1}, x_{2}, k_{3}\right)$ are the rotation-induced pressure and streamwise vorticity in the Fourier space, respectively, and $k_{1}$ and $k_{3}$ represent the streamwise and spanwise wavenumbers, respectively. The solution to Eq. (8) can be obtained analytically, ${ }^{16}$ viz.,

$$
\hat{p}_{r}=\frac{2 \rho \Omega}{h} \int_{-h}^{h} G\left(k, x_{2}, \bar{x}_{2}\right) \hat{\omega}_{1}\left(k_{1}, \bar{x}_{2}, k_{3}\right) \mathrm{d} \bar{x}_{2} .
$$

Here, the Green function $G\left(k, x_{2}, \bar{x}_{2}\right) \operatorname{reads}^{16}$

$$
G\left(k, x_{2}, \bar{x}_{2}\right)= \begin{cases}-\frac{\cosh \left[k\left(\bar{x}_{2}-h\right)\right] \cosh \left[k\left(x_{2}+h\right)\right]}{2 k h \cosh (k h) \sinh (k h)}, & k \neq 0 \text { and } x_{2}<\bar{x}_{2} \\ -\frac{\cosh \left[k\left(\bar{x}_{2}+h\right)\right] \cosh \left[k\left(x_{2}-h\right)\right]}{2 k h \cosh (k h) \sinh (k h)}, & k \neq 0 \text { and } x_{2}>\bar{x}_{2}, \\ \frac{\left|\bar{x}_{2}-x_{2}\right|}{2 h}, & k=0\end{cases}
$$

where $k=\left(k_{1}^{2}+k_{3}^{2}\right)^{\frac{1}{2}}$. According to Eq. (10), the value of $G$ is negative for $k \neq 0$ such that the values of $p_{r}^{\prime}$ and $\omega_{1}^{\prime}$ exhibit a negative correlation $(k=0$ corresponds to the mean pressure $\left\langle p_{r}\right\rangle$ and mean vorticity $\left.\left\langle\omega_{1}\right\rangle\right)$. Because $p_{r}^{\prime}$ is the dominant component of $p^{\prime}$ at $R o_{\tau}=150$, this explains the interesting observation from Fig. 2(a) that the positive and negative extrema of $p^{\prime}$ well overlap the centers of TGL vortices rotating in the counterclockwise and clockwise directions, respectively. Furthermore, the magnitude of $G$ at specific values of $\bar{x}_{2} / h$ and $x_{2} / h$ decreases monotonically as $k$ increases, indicating that the integral transform based on kernel function $G$ tends to shift the spectra of $\omega_{1}^{\prime}$ toward smaller wavenumbers.

To verify this conclusion of the spectral analysis, it is useful to compare the pre-multiplied streamwise spectra $\Phi_{1}\left(\omega_{1}^{\prime}\right)$ and $\Phi_{1}\left(p_{r}^{\prime}\right)$. Following the literature, ${ }^{17-19}$ we show premultiplied spectra (instead of the spectra) to clearly demonstrate the contributions from different wavelengths in a semilogarithmic coordinate. Here, the pre-multiplied streamwise spectrum $\Phi_{1}\left(\phi^{\prime}\right)$ of an arbitrary fluctuating flow quantity $\phi^{\prime}$ is defined as $\Phi_{1}\left(\phi^{\prime}\right)=k_{1} E_{1}\left(\phi^{\prime}\right)$, where the streamwise spectrum $E_{1}\left(\phi^{\prime}\right)$ is calculated as the Fourier transform of the streamwise two-point correlation $R_{1}\left(\phi^{\prime}\right)$, viz.,

$$
E_{1}\left(\phi^{\prime}\right)=\frac{1}{\pi} \int R_{1}\left(\phi^{\prime}\right) e^{-\mathrm{i} k_{1} r_{1}} \mathrm{~d} r_{1} .
$$

Here, $\mathrm{i}=\sqrt{-1}$, the two-point correlation function is defined as $R_{1}\left(\phi^{\prime}\right)=\left\langle\phi^{\prime}\left(x_{1}, x_{2}, x_{3}, t\right) \phi^{\prime}\left(x_{1}+r_{1}, x_{2}, x_{3}, t\right)\right\rangle$, and $r_{1}$ is the streamwise separation between two points.

Figure 5 compares $\Phi_{1}\left(\omega_{1}^{\prime}\right), \Phi_{1}\left(p_{r}^{\prime}\right), \Phi_{1}\left(u_{2}^{\prime}\right)$, and $\Phi_{1}\left(u_{3}^{\prime}\right)$ at $R o_{\tau}=150$ in the plane located at $x_{2} / h=-0.5$, where TGL vortices are populated [see Fig. 2(a)]. In Fig. 5, the value of $\Phi_{1}\left(\phi^{\prime}\right)$ is normalized by $\left\langle\phi^{\prime} \phi^{\prime}\right\rangle$ such that the area below each spectrum curve remains unity. As is evident from Fig. 5, $\Phi_{1}\left(p_{r}^{\prime}\right)$ peaks at a smaller wavenumber $k_{1} h$ than does $\Phi_{1}\left(\omega_{1}^{\prime}\right)$, which confirms the previous analysis that the Green function $G$ shifts the pre-multiplied spectra of $\omega_{1}^{\prime}$ toward smaller wavenumbers. The peaks of $u_{2}^{\prime}$ and $u_{3}^{\prime}$ are close to each other and are between those of $p_{r}^{\prime}$ and $\omega_{1}^{\prime}$.

Figure 6(a) further compares the streamwise premultiplied spectra of $p_{r}^{\prime}$ at various rotation numbers. It is evident from Fig. 6(a) that $\Phi_{1}\left(p_{r}^{\prime}\right)$ shifts progressively toward smaller wavenumbers as the rotation number increases, which is consistent with our previous observation of the scale trend of TGL vortices. ${ }^{7}$

Figure 6(b) shows the effect of the rotation number on the streamwise pre-multiplied spectra of $u_{2}^{\prime}$. As demonstrated in Fig. 6(b), the peak of $\Phi_{1}\left(u_{2}^{\prime}\right)$ in a non-rotating channel $\left(R o_{\tau}=0\right)$ occurs at $k_{1} h=4.5$. This peak is contributed exclusively by the convection-induced pressure fluctuations $p_{c}^{\prime}$ (because in a nonrotating channel, $\left.p_{r}^{\prime} \equiv 0\right)$. Once the system rotation is imposed on the flow, at a low rotation number $R o_{\tau}=7.5$, the peak at $k_{1} h=4.5$ is still present, but its magnitude is smaller than that at $R o_{\tau}=0$. A secondary peak occurs at a much smaller wavenumber $k_{1} h=0.38$ (at $R o_{\tau}=7.5$ ), which is close to the peak location $\left(k_{1} h=0.31\right)$ of $p_{r}^{\prime}$ shown in Fig. 6(a), indicating that this secondary peak is a consequence of the rotationinduced pressure fluctuations $p_{r}^{\prime}$. The peak of $\Phi_{1}\left(u_{2}^{\prime}\right)$ for $p_{c}^{\prime}$ diminishes rapidly as the rotation number becomes greater than 15 , and the spectrum shifts toward smaller wavenumbers as the rotation number further increases. The pre-multiplied spectrum of $u_{3}^{\prime}$ also shifts toward smaller wavenumbers but is not shown to keep the discussion concise. The effects of system rotation on $u_{2}^{\prime}$ and $u_{3}^{\prime}$ are consistent with that on $p_{r}^{\prime}$ shown in Fig. 6(a), indicating that large-scale velocity fluctuations $u_{2}^{\prime}$ and $u_{3}^{\prime}$ correlate with the rotation-induced pressure fluctuation $p_{r}^{\prime}$.

The interaction between $p_{r}^{\prime}$ and $u_{2}^{\prime}$ or $u_{3}^{\prime}$ is nonlinear, which can be further understood through the transport equation of Reynolds stresses [Eq. (7.4) in the work of Yang and Wang ${ }^{7}$ ]. In our previous study, ${ }^{7}$ it was shown that the budget balance

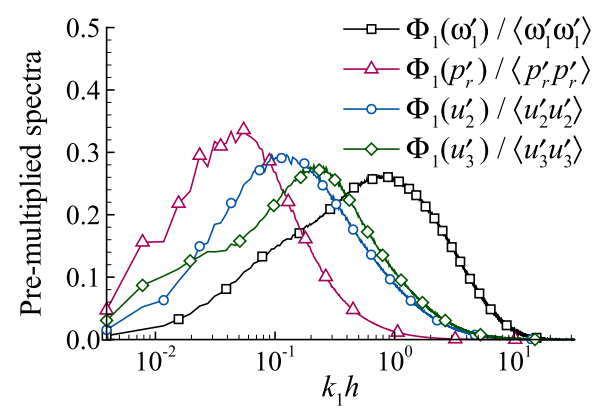

FIG. 5. Pre-multiplied streamwise spectra of $\omega_{1}^{\prime}, p_{r}^{\prime}, u_{2}^{\prime}$, and $u_{3}^{\prime}$ in the plane located at $x_{2} / h=-0.5$ for $R o_{\tau}=150$. 


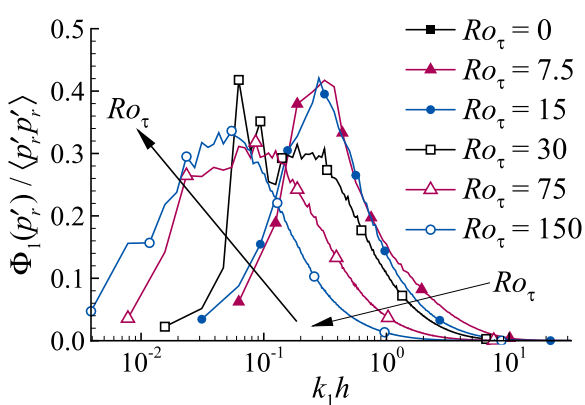

(a)

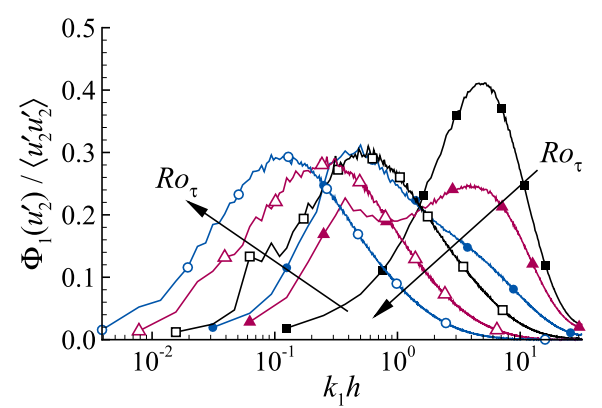

(b)

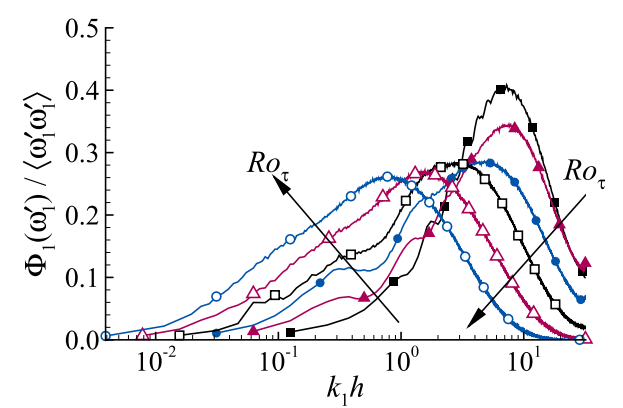

(c)

of $\left\langle u_{2}^{\prime} u_{2}^{\prime}\right\rangle$ is dominated by two counteracting terms, i.e., the pressure term $G_{22}$ and the Coriolis terms $C_{22}=4 \Omega\left\langle u_{2}^{\prime} u_{3}^{\prime}\right\rangle$ (and similarly, the budget balance of $\left\langle u_{3}^{\prime} u_{3}^{\prime}\right\rangle$ is dominated by $G_{33}$ and $C_{33}=-C_{22}$ ) in a fast streamwise-rotating flow. Here, the pressure term is defined as $G_{i j}=-\left\langle u_{i}^{\prime} \partial p^{\prime} / \partial x_{j}+u_{j}^{\prime} \partial p^{\prime} / \partial x_{i}\right\rangle / \rho$.

Large-scale fluctuating streamwise vorticity $\omega_{1}^{\prime}$ is correlated with velocity fluctuations (i.e., $u_{2}^{\prime}$ and $u_{3}^{\prime}$ ) of the TGL vortices, as is clear from the definition equation of $\omega_{1}^{\prime}$. Figure 6(c) compares the streamwise pre-multiplied spectra of $\omega_{1}^{\prime}$ at various rotation numbers. Similar to $\Phi_{1}\left(p_{r}^{\prime}\right)$ and $\Phi_{1}\left(u_{2}^{\prime}\right)$ demonstrated in Figs. 6(a) and 6(b), respectively, $\Phi_{1}\left(\omega_{1}^{\prime}\right)$ also shifts toward smaller wavenumbers as the rotation number increases. Although the magnitude of $\omega_{1, \text { rms }}$ is insensitive to the rotation number [see Fig. 4(a)], the results shown in Fig. 6(c) indicate that as the rotation number increases, TGL vortices become increasingly elongated in the streamwise direction, which tends to reduce the dominant wavenumbers of $\omega_{1}^{\prime}$.

To conclude, in order to investigate the influence of streamwise system rotation on the size, strength, and characteristic wavelength of TGL vortices, we decomposed the pressure field linearly into a rotation-induced component and a convection-induced component ( $p_{r}^{\prime}$ and $p_{c}^{\prime}$, respectively), governed by two independent Poisson equations. By contrast, in a non-rotating channel flow, pressure fluctuations are governed by one single Poisson equation, for the convection-induced component only. The Coriolis force acts on the pressure field as a source term in the Poisson equation that governs the value of $p_{r}^{\prime}$. It is discovered that the streamwise system rotation functions as a linear amplifier. In response to a fast system rotation, the system rotation $(\Omega)$ converts streamwise vorticity fluctuations $\omega_{1}^{\prime}$ into rotation-induced pressure fluctuations $p_{r}^{\prime}$ through the source term of a Poisson equation. Owing to the Laplace differential operator of the Poisson system, both the amplitude and the wavenumber of $p_{r}^{\prime}$ are modulated by the streamwise system rotation imposed.
FIG. 6. Pre-multiplied streamwise spectra of (a) $p_{r}^{\prime}$, (b) $u_{2}^{\prime}$, and (c) $\omega_{1}^{\prime}$ in the plane located at $x_{2} / h=-0.5$ for various rotation numbers.
It is discovered that there exists a critical rotation number, at which the effect of rotation-induced pressure fluctuations outweighs that of the convection-induced pressure fluctuations. TGL vortices are not observed if the rotation number is very low. This is because the pressure is dominated by the convection-induced part at a low rotation number such that the pre-multiplied streamwise spectra of the velocity fluctuations peak at streamwise wavenumber $k_{1} h=4.5$ [as indicated by $\left.\Phi_{1}\left(u_{2}^{\prime}\right)\right]$. However, at a relatively high rotation number, a secondary peak occurs at a much smaller wavenumber $k_{1} h=0.38$, indicating that this secondary peak is a consequence of the rotation-induced pressure fluctuations $p_{r}^{\prime}$. Furthermore, the peak of $\Phi_{1}\left(u_{2}^{\prime}\right)$ for convection-induced pressure fluctuations $p_{c}^{\prime}$ diminishes rapidly as the rotation number becomes greater than 15 , and the spectrum shifts toward smaller wavenumbers as the rotation number continues to increase. As such, the fundamental difference between streaky structures and TGL vortices is revealed through this study (by precisely identifying their characteristic wavenumbers, $k_{1} h=4.5$ and 0.38 ), which are two different types of turbulence structures in a wall-bounded rotating flow, dominated by convection and streamwise-rotation, respectively.

The authors would like to thank the Western Canada Research Grid (WestGrid) for providing access to supercomputing and storage facilities. Research funding from the Natural Sciences and Engineering Research Council (NSERC) of Canada to B.-C. Wang is gratefully acknowledged.

${ }^{1}$ T. Weller and M. Oberlack, "DNS of a turbulent channel flow with streamwise rotation-Investigation on the cross flow phenomena," in Direct and Large-Eddy Simulation VI, edited by E. Lamballais, R. Friedrich, B. J. Geurts, and O. Métais (Springer, 2006), pp. 241-248.

${ }^{2} \mathrm{~T}$. Weller and M. Oberlack, "DNS of a turbulent channel flow with streamwise rotation-Study of the reverse effect of the cross flow," Proc. Appl. Math. Mech. 6, 553-554 (2006).

${ }^{3}$ M. Oberlack, W. Cabot, B. A. P. Reif, and T. Weller, "Group analysis, direct numerical simulation and modelling of a turbulent channel flow with streamwise rotation," J. Fluid Mech. 562, 383-403 (2006). 
${ }^{4} \mathrm{H}$. Wu and N. Kasagi, "Effects of arbitrary directional system rotation on turbulent channel flow," Phys. Fluids 16, 979-990 (2004).

${ }^{5}$ I. Recktenwald, T. Weller, W. Schröder, and M. Oberlack, "Comparison of direct numerical simulations and particle-image velocimetry data of turbulent channel flow rotating about the streamwise axis," Phys. Fluids 19, 085114 (2007).

${ }^{6}$ Y.-T. Yang, W.-D. Su, and J.-Z. Wu, "Helical-wave decomposition and applications to channel turbulence with streamwise rotation," J. Fluid Mech. 662, 91-122 (2010).

${ }^{7}$ Z. Yang and B.-C. Wang, "Capturing Taylor-Görtler vortices in a streamwise-rotating channel at very high rotation numbers," J. Fluid Mech. 838, 658-689 (2018).

${ }^{8}$ W. S. Saric, "Görtler vortices," Annu. Rev. Fluid Mech. 26, 379-409 (1994).

${ }^{9}$ J. M. Floryan, "Görtler instability of boundary layers over concave and convex walls," Phys. Fluids 29, 2380-2387 (1986).

${ }^{10}$ R. Kristoffersen and H. I. Andersson, "Direct simulations of low-Reynoldsnumber turbulent flow in a rotating channel," J. Fluid Mech. 256, 163-197 (1993).
${ }^{11}$ O. Grundestam, S. Wallin, and A. Johansson, "Direct numerical simulations of rotating turbulent channel flow," J. Fluid Mech. 598, 177-199 (2008).

${ }^{12}$ J. Jiménez and A. Pinelli, "The autonomous cycle of near-wall turbulence," J. Fluid Mech. 389, 335-359 (1999).

${ }^{13}$ Y. Hwang and C. Cossu, "Self-sustained process at large scales in turbulent channel flow," Phys. Rev. Lett. 105, 044505 (2010).

${ }^{14}$ Y. Hwang and Y. Bengana, "Self-sustaining process of minimal attached eddies in turbulent channel flow," J. Fluid Mech. 795, 708-738 (2016).

${ }^{15}$ R. A. Antonia and J. Kim, "Low-Reynolds-number effects on near-wall turbulence," J. Fluid Mech. 276, 61-80 (1994).

${ }^{16} \mathrm{~J}$. Kim, "On the structure of pressure fluctuations in simulated turbulent channel flow," J. Fluid Mech. 205, 421-451 (1989).

${ }^{17}$ J. C. del Álamo and J. Jiménez, "Spectra of the very large anisotropic scales in turbulent channels," Phys. Fluids 15, L41-L44 (2003).

${ }^{18} \mathrm{~S}$. Hoyas and J. Jiménez, "Scaling of the velocity fluctuations in turbulent channels up to $\operatorname{Re}_{\tau}=2003$," Phys. Fluids 18, 011702 (2006).

${ }^{19}$ V. Avsarkisov, S. Hoyas, M. Oberlack, and J. P. García-Galache, "Turbulent plane Couette flow at moderately high Reynolds number," J. Fluid Mech. 751, R1 (2014). 